\title{
QUALITY AND POPULATION
}

George Rubin, Michael Frommer and

Johanna Westbrook,

Epidemiology and Health Services Evaluation Branch, NSW Health Department

This article is based on a presentation by George Rubin at the Workshop on Quality Assurance of the International Society for Quality Assurance in Health Care at the Howard Florey Institute, Melbourne, Victoria, March 27, 1993.

" Erom the catwalks of healthcare fashion, outcomes research emerges as the star of the show. While the benefits of clinical activity have motivated doctors for generations, the new imperative is to combine these with the consumerist agenda - in effect, to put professional standards into consumer values." These glittering lines from a recent Lancet editorial herald new directions in health care.

Meantime, governments and health organisations strive to better assess, in health terms, the effectiveness of resources devoted to health services. Each year in Australia we spend around $\$ 32$ billion. What do we achieve in terms of improving the health status and wellbeing of the population? Could these resources be allocated in a different way to obtain greater health benefits?

While we have become preoccupied with improving the quality of care there is a major gap in the way we are going about this. The World Health Organisation (WHO) defines quality as improving the outcome of all health care in terms of health, functional ability, patient wellbeing and consumer satisfaction. The quality assurance process deals with three elements - structure, process and outcome. A health outcome is a change in the health of an individual or population which is attributable to interventions.

The deficiency that we want to draw to your attention is a failure to link outcomes on the one hand with structure and process on the other. Most quality assurance activities have focused on only structure or process standards, with no clear relation to outcome. Indeed, whether these activities improve health benefits remains unclear and there is a growing recognition that many health interventions have not been evaluated and their effectiveness is not known.

Moreover, quality is not just about obtaining the best outcomes regardless of the cost of the structure and process needed to do this. Health resources are most appropriately used for interventions which provide the best value for money.

We have the methods to make this link between outcomes and the quality of health interventions. We have been using epidemiologic methods for a long time to describe and analyse health-related phenomena in populations. They have enormous potential in quality assurance to quantify how the structure and process of health interventions relates to their outcomes.

Epidemiology should underpin the key elements of health service management:

뭄

information analysis and policy formulation; planning and program development; program delivery; and

evaluation.

Epidemiology has been going through a long, slow transition from its historic association with the old style of public health (communicable diseases, rats and drains) to the realisation that it is crucial to good decision making in health and hence to good health services management. assurance requires a reorientation of thinking in five key directions.

1. Implement an outcomes focus in health systems We are beginning to make good progress on this in Australia. The Commonwealth has introduced health outcomes into the Medicare agreements. States will be required to contribute to the development of outcomes-based accountability systems. The National Health and Medical Research Council (NHMRC) has established a Quality of Care Committee to enhance this process and develop mechanisms for establishing appropriate practice guidelines.

NSW has a Health Outcomes Program. Under this program epidemiologists are working with clinicians, consumers, managers and health economists. The starting point is to ask what is the purpose of a particular clinical or public health service and then to agree on measurable health outcome indicators that fit with the explicit purpose of the service. The next steps are to establish data systems to collect, monitor and feed back information on the indicators, and for health service providers to use this information to improve the structure, process and outcomes of their services.

How do we make all this happen? In NSW we will shortly announce a series of demonstration projects which exemplify this sequence in cardiovascular disease, critical care, asthma, diabetes, immunisation and Aboriginal health. This leads me to the second direction.

\section{Incorporate outcome measures into population health planning}

Measurable health outcome objectives should increasingly be included in health department and local health service plans. This is already happening to a greater or lesser extent in NSW, Victoria, Tasmania and Western Australia. In NSW, where increasing emphasis on outcome measurement is apparent, the corporate plan is used as the basis for performance agreements between the area chief executives and the Director-General. Incentives to adopt outcomes approaches at the service level and involving consumers will undoubtedly increase as local information systems improve.

\section{Foster links with health economics}

Earlier the issue of value for money was raised. As well as indicators we need information on the resources involved in achieving the outcomes. In the lineup of people involved in the NSW outcomes program have been included health economists. A central plank in our outcomes approach is to involve health economists at the beginning of the process.

\section{Improve information systems}

Current developments in information technology provide a seamless information capture mechanism extending from points of clinical contact to aggregated data at the hospital, area and Statewide level. The developments will enable us to effect the link between health interventions and their outcomes at the service and population levels. Examples of information systems with these capacities already exist.

Under the NSW Quality Assurance Program clinicians are developing local PC-based outcome monitoring systems to improve their services in asthma, cardiovascular disease and obstetric care.

To get epidemiology into the mainstream of quality 
$P_{s}$ fessor James S. Lawson, Professor and Head of the School of Health Services Management at the University of NSW, has prepared the following public health items from the literature.

\section{DISABILITY AMONG IMMATURE INFANTS}

Neo-natal intensive care has been described as 'perhaps the most successful of all medical technologies'. In terms of improving the chance of survival this may be so, but the rate of disabilities among the survivors is high as has been shown by a comprehensive survey involving nearly 100,000 infants in the United Kingdom. About 3.5 of 1,000 of these births were before 29 weeks of gestation. Half the babies survived to be discharged from the nursery. At four years, 93 per cent of the premature infants were still alive. Only 35 per cent of those four-year-olds were within normal limits. Around 29 per cent had mild disability, 13 per cent a moderate disability and 23 per cent were severely disabled. The severe disablements included cerebral palsy, blindness, severe hearing loss and intellectual handicap. A number of babies had multiple disabilities. An important finding was that the incidence of disability increases with declining gestational age of the babies.

Johnson A, Townshend P, Yudkin P et al. Functional abilities at age 4 years of children born before 29 weeks of gestation. $B r$ Med J, 1993; 306:1715-1718.

\section{NEVER DISMISS WHAT A PATIENT TELLS YOU}

When 90-year-old Burt Adams was admitted to hospital he asked the staff to let his mother know. The doctors thought he must be senile. In fact, his mother Daisy, at 113 years, is the oldest woman in Britain. (The names are fictitious.)

Editorial. Br Med J, 1993; 307:48-49.

\section{SEX, PREGNANCY, HORMONES AND MELANOMA}

Many questions remain unanswered about the relationship between melanoma (the most rapidly increasing Australian cancer) and the hormonal environment. Several conclusions can be made within the current state of knowledge. First, there is no evidence that the use of oestrogens, either as oral contraceptives or hormone replacement therapy, has a role in the aetiology of melanoma. Second, women have a survival advantage over men that could be due to the inhibitory effect of normal oestrogens in the growth of melanoma. Third, prescribed oestrogens do not promote progression of the disease in patients with melanoma, therefore women who have been treated for melanoma can safely use hormonal supplements. Last, pregnancy seems to carry no adverse effect on survival after treatment for melanoma. (However, patients with thick melanomic lesions are advised to delay pregnancy for two to three years as this is when they are at the greatest risk of relapse).

Jatoi I and Gore ME. Sex, pregnancy, hormones and melanoma. $\mathrm{Br}$ Med $J, 1993 ; 307: 2-3$

\section{BREAST-FEEDING REDUCES RISK OF BREAST CANCER}

A large British study has confirmed that breast-feeding is associated with a statistically significant decreased risk of breast cancer. The risk of breast cancer falls with increased duration of breast-feeding, and with the number of babies breast-fed. However, breast-feeding each baby for longer than three months confers no additional benefits.

United Kingdom National Case-Control Study Group. Breast feeding and risk of breast cancer in young women. Br Med J, 1993; 307:17-20.

\section{PRIVILEGE AND HEALTH - WHAT IS THE CONNECTION?} Socio-economic status is a powerful determinant of health. In current jargon, socio-economic status refers to a mix of factors that shape a person's relative social advantage. It is usually gauged by income, education, profession or some combination of the three. But no-one knows exactly which factors determine health, much less how they do so. This is a crucial issue as we know that in Australia the premature death rates among the highest social categories are about half those of the lowest social categories. The differences do not seem to be simply a matter of the privileged having better access to health care. So closely does socio-economic status co-relate with health that it confounds the interpretation of much clinical research. For example, studies of the effect of passive smoking on childhood asthma are uninterpretable unless an attempt is made to control for socio-economic status. Until more specific knowledge is

\section{Quality and population}

$\checkmark$ Continued from page 137

\section{Improve epidemiologic capability}

There is much to be done requiring epidemiologic capability. This is not to say that we necessarily need more epidemiologists, however, we do need to get clinicians, managers, and even consumers thinking epidemiologically.

Training in, and practice of, epidemiology has expanded dramatically in Australia over the past five years. There has been a proliferation of university public health courses offering epidemiology as a key subject. The Faculty of Public Health Medicine has formed to improve training of doctors in the practice of epidemiology and public health. National and State programs have developed to train health professionals, both medical and non-medical, to apply epidemiologic principles to improving health services. Public health networks staffed with young epidemiologists are developing in most States.

NSW has established Statewide epidemiologic expertise with a central unit and a network of public health units. A training program encourages health professionals into public health and epidemiologic practice and links are being forged between the public health network, health services management and clinical practice.

We started by identifying the major gap between the structure and process preoccupations of current quality assurance thinking on the one hand, and health outcomes on the other. We have the means to close this gap, and we are already doing it. Optimistic that what is already happening will snowball over the next 12 months, we predict that health outcomes will remain the star on the catwalks of healthcare fashion. We will be well on the way to closing feedback loops between outcomes and the quality of services. 